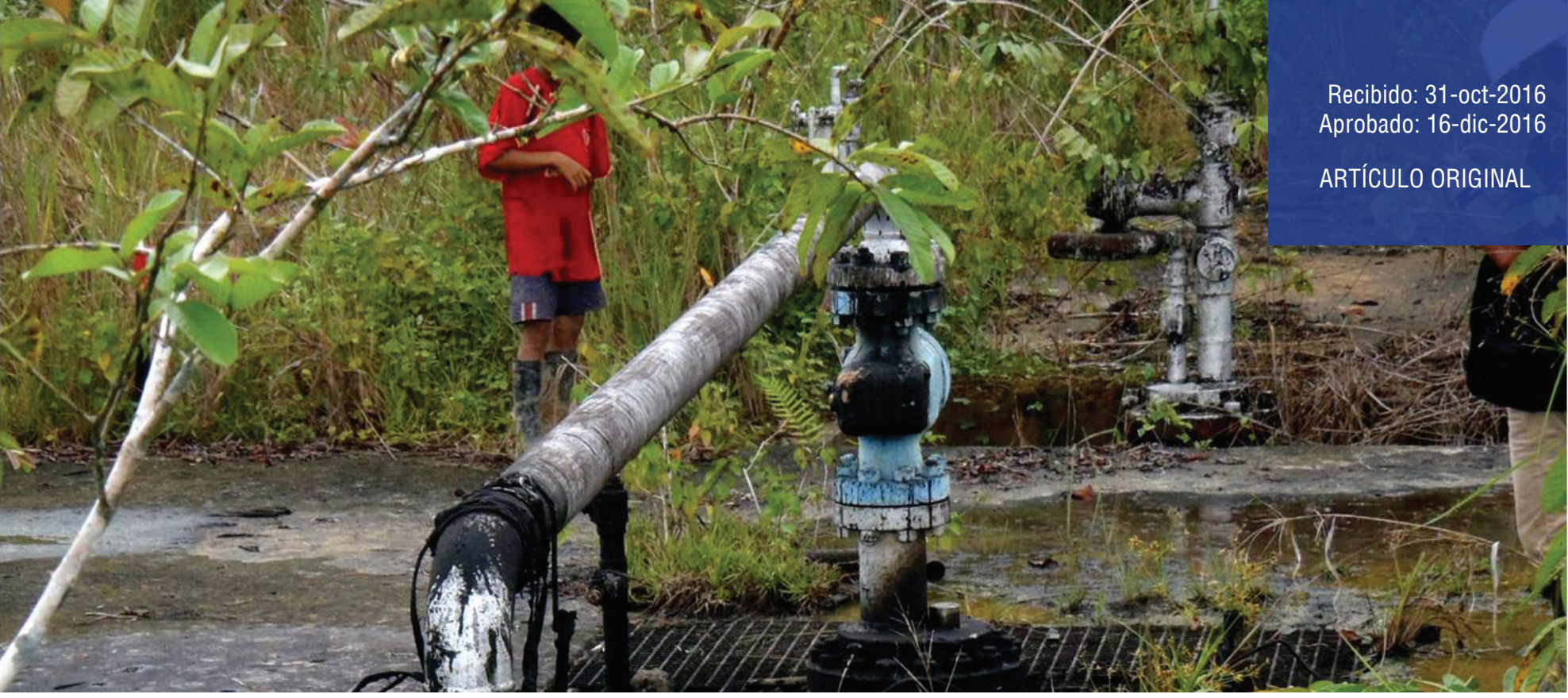

\title{
Generación de indicadores de pasivos ambientales y sociales para el subsistema de inteligencia de estadísticas aplicado a las actividades hidrocarburíferas
}

\author{
Generation of indicators of environmental and social liabilities for the subsystem of \\ intelligence of statistics applied to activities hydrocarbon
}
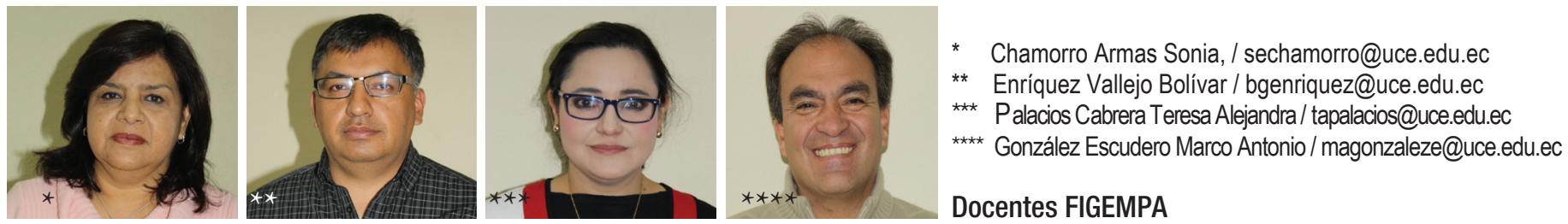

Resumen

El presente estudio permitió la generación de indicadores de pasivos ambientales y sociales con el cual se mide el avance de la gestión de las actividades hidrocarburíferas y potenciar la remediación ambiental, mediante la aplicación de instrumentos de política pública. La sobreexplotación de servicios ambientales causa afectaciones de depredación y/o contaminación a los ciclos naturales, degradando el entorno en el que habitan las comunidades de flora, fauna y humanas, provocando una vulneración de sus derechos. Se utilizó una metodología descriptivapropositiva, con un enfoque holístico, que permitió desarrollar cinco indicadores basados en: la fuerza motriz (número de derrames, piscinas y fosas); presión (número de reclamos por fuentes de contaminación); estado (medidas de satisfacción implementadas por derrames, piscinas y fosas); impacto (número de convenios por actividad hidrocarburífera) y respuesta (número de derrames, piscinas y fosas remediadas por Autoridad Ambiental), al principio de la Reparación Integral. Propuesta que permitirá contextualizar el estado de la gestión de los pasivos ambientales en el Ecuador. Los indicadores propuestos en el sector hidrocarburífero a la afectación por contaminación, desarrollará políticas de explotación de recursos de manera sustentable.

Palabras clave: pasivos ambientales; indicadores socio-ambientales; sector hidrocarburífero; ciclo natural, contaminación; remediación; derechos

\section{Abstract}

This study allowed the generation of indicators of environmental and social liabilities which is measured the progress of the management of hydrocarbon activities and enhance the environmental remediation, through the application of instruments of public policy. Overexploitation of environmental services causes effects of predation or pollution to natural cycles, degrading the environment in which inhabit communities of flora, fauna and human, causing an infringement of their rights. We used a methodology descriptive and purposeful with a holistic approach which allowed to develop five indicators based on the power supply (number of spills, pools and ditches), pressure (number of claims by sources of pollution), State (satisfaction measures implemented by spills, pools and ditches) impact (number of conventions by hydrocarbon activity) and response (spills, pools and pits remediated by environmental authority number), at the beginning of the Integral service. Proposal that will allow contextualize the State of the management of the passive environmental in the Ecuador. The indicators proposed in the hydrocarbon (PRAS) to the affected sector by pollution, develop policies of exploitation of resources in a sustainable way. 


\section{Marco teórico}

Contexto de la actividad hidrocarburífera en el Ecuador

La actividad hidrocarburífera de petróleo y gas se desarrolla en etapas: "upstream" que son explotación y exploración y "downstram" sector transporte, distribución y comercialización (Campodónico, abril, 2007, págs. 7, 9). (PNUMA Oficina Regional para América Latina y el Caribe, 2008, pág. 14). Este sector es responsable de emisiones de gases de efecto invernadero (GEI), a la atmósfera que repercuten en forma negativa a nivel mundial, regional y local. El sector de transporte también es fuente de emisiones tales como material particulado, plomo, óxidos de nitrógeno $\left(\mathrm{NO}_{\mathrm{x}}\right)$, óxidos de azufre (SO) y compuestos orgánicos volátiles (COV), que ocasionan problemas ambientales y de salud a escala local y, a menudo, regional; según los inventarios de emisiones estimadas, las fuentes móviles son responsables de la mayor parte de la contaminación atmosférica en las áreas urbanas de la región. Además de material particulado, los vehículos automotores son también importantes emisores de óxidos de nitrógeno, óxidos de azufre, ozono y monóxido de carbono, que tienen graves repercusiones en la salud de la población (ONU-CEPAL, op. cit., pág. 51).

\section{El ámbito de estudio: La deuda o pasivo ambiental pro- puesta conceptual}

Es pertinente entender cómo funcionan los sistemas sociales y jurídicos que regulan el tratamiento por los daños realizados a la naturaleza, entendiendo por sistemas sociales a sistemas inventados, ideados artificialmente, construidos por el hombre (Navarro. C. 2001); en los sistemas sociales es habitual el conflicto entre políticas a largo y corto plazo, ello condiciona la toma de decisiones, en función de un interés temporal más inmediato 0 más lejano (Navarro Cid, op. cit., págs. 44, 45).

\section{El pasivo ambiental y la extensión del principio de repara- ción integral al ámbito biofísico en Ecuador.}

Sukhdev \& Berghöfer (2007) anotan que para incrementar el bienestar humano, no basta con el crecimiento económico, se requiere considerar los costos, el capital natural consumido para sostener ese crecimiento y muchos beneficios de la naturaleza encaminados al bienestar humano que se pierden en el camino de la política económica convencional y en las prácticas empresariales. Una manera sistemática de evaluar tales daños es contemplar la naturaleza en términos de servicios ambientales; los diversos beneficios que obtenemos de la naturaleza para nuestro bienestar; la pérdida de biodiversidad y la degradación de los servicios del ecosistema continúan a pesar de los decisivos esfuerzos de planificadores, administradores. Un modelo de interrelación entre la economía y los ecosistemas que refleja este no pago por los servicios ambientales es realizado por Gerardo Barrantes, Director del Instituto de Políticas para la Sostenibilidad de Costa Rica (IPS).

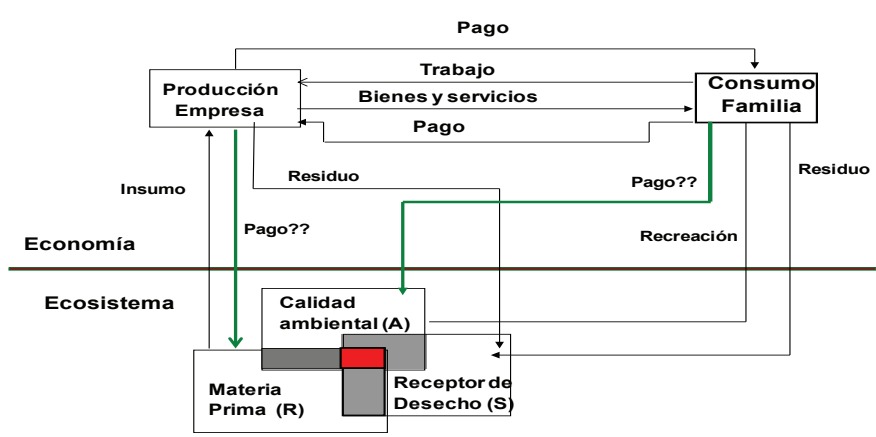

Figura 1 Relaciones entre la economía y los ecosistemas.

Fuente: (Barrantes, G. "Metodología para la Evaluación Económica de Daños Ambientales"

Instituto de Políticas para la Sostenibilidad (Costa Rica) www.ips.or.cr; 1/29/2014).

"Pasivo Ambiental" se define como "[...] aquellos daños ambientales $y / 0$ impactos ambientales negativos no reparados o restaurados respectivamente, 0 que han sido intervenidos previamente de forma inadecuada y continúan estando presentes en el ambiente, constituyendo un riesgo para cualquiera de sus componentes, generados por un proyecto 0 una actividad productiva o económica en general" (Ministerio del Ambiente, 2012, pág. 4).
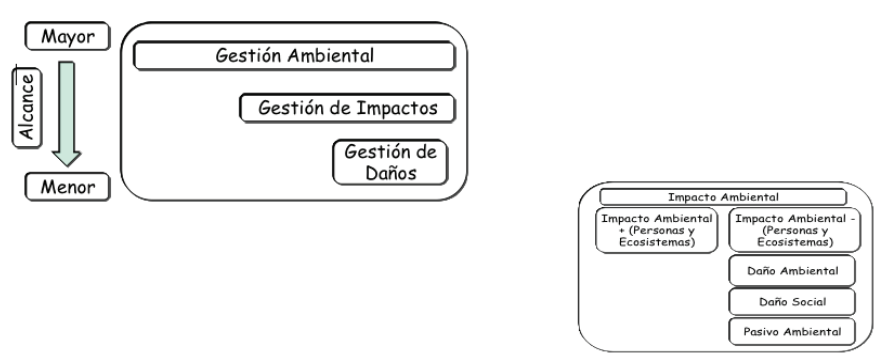

Figura 2. Gestión Ambiental y la Ubicación de la GPA

Desde un punto de vista holístico, el principio de reparación integral con enfoque ecocéntrico, puede considerarse como una herramienta técnica política que permite saldar la deuda ambiental-pasivo- ambiental, contraída por la interacción de las actividades humanas en el proceso de desarrollo que causan cambios de estado en el sistema Tierra, por sobrepasar la capacidad del ecosistema para producir servicios y reciclar desechos. Es decir, la Reparación Integral es un instrumento técnico legal que permite alcanzar el "pago" de la deuda ambiental con la implementación de procesos que equilibren las relaciones hombre-ambiente, a fin de resarcir los derechos vulnerados de la naturaleza.

\section{Universo y muestra}

La muestra comprende el territorio que ocupan las actividades 
hidrocarburíferas en el Ecuador (Atlas Geográfico del Ecuador. 2013) sobre las fuentes de contaminación al agua, aire y suelo. Para estos mapas hay que tomar en cuenta que todavía no se dispone de la medición de emisiones y descargas contaminantes totales (IGM, Senplades, 2013, pág. 266).

Se identifican los sectores productivos vinculados con los asentamientos humanos según Senplades (2013) en el Plan Nacional del Buen vivir 2013-2017.

Las fuentes de contaminación al aire en el Ecuador se presentan en las centrales térmicas de generación eléctrica, refinerías de petróleo, contaminación por ruido referidas a las urbes, emisiones vehiculares y áreas industriales (IGM, Senplades, 2013, págs. 268, mapa 16 fuentes de contaminación del aire).

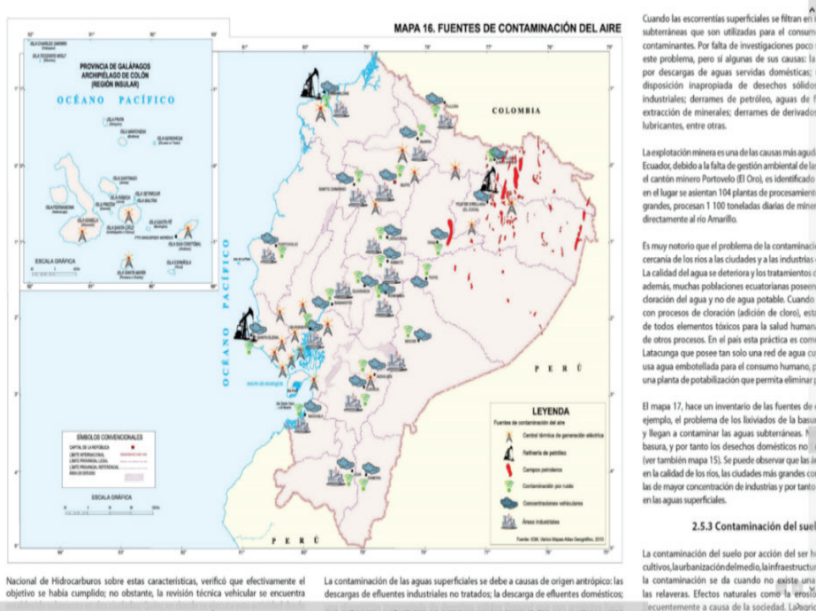

Figura 3. Mapa de ubicación de las principales fuentes de contaminación antropogénicas al aire en el Ecuador (IGM, 2013).

Fuente: (IGM, Senplades, 2013, págs. 268, mapa 16 fuentes de contaminación del aire).

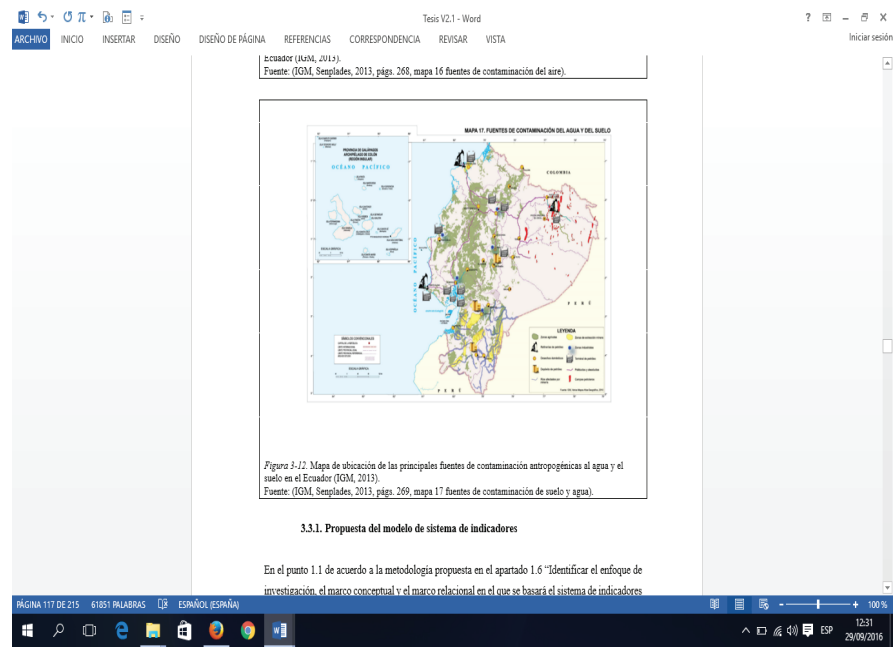

Figura 4. Mapa de ubicación de las principales fuentes de contaminación antropogénicas al agua y el suelo en el Ecuador (IGM, 2013).

Fuente: (IGM, Senplades, 2013, págs. 269, mapa 17 fuentes de contaminación de suelo y agua).

\section{Propuesta del modelo de sistema de indicadores}

La metodología planteada propone Identificar el enfoque de investigación, el marco conceptual y el marco relacional, en el que se basará el sistema de indicadores y que empleará el enfoque sistémico, debido a que el ámbito analizado es un fenómeno holístico que integra varias dimensiones: económica, ecológica y social, por lo que, no puede ser comprendido desde un enfoque reduccionista, ya que tiene varias características que se enmarcan dentro del enfoque de sistemas en el marco conceptual de la ciencia del sistema Tierra y la teoría general de sistemas y de Miller, y que sirve como base de análisis relacional para la propuesta del modelo de indicadores e interpretación de los mismos. Plantea el concepto de desarrollo sustentable con el fin de llegar al estudio del ámbito pasivo ambiental. Además busca generar el modelo conceptual del sistema de indicadores e identificar el o los marcos ordenadores y jerárquicos de presentación de los mismos. Hay que tomar en cuenta que para la generación de este modelo, es importante conocer el objetivo del sistema que es, medir el grado de deuda o pasivo y las interacciones humanas a través de la aplicación del principio de la reparación integral; por lo que se considera que la deuda 0 pasivo ambiental es una disrupción del equilibrio en el sistema Tierra, cuando las actividades económicas y humanas no respetan la constitución natural o real de los sistemas que funcionan en el planeta, que sería el modelo sostenible y en equilibrio. La deuda se genera en un estado de alejamiento del equilibrio.

\section{Resultados}

\section{Tabla 1}

Propuesta de marco ordenador fusionado con los elementos del principio de reparacióncon enfoque ecocéntrico para el sistema de indicadores de pasivos ambientales propuesto.

\begin{tabular}{|c|c|c|c|}
\hline & $\begin{array}{l}\text { Medida de } \\
\text { Reparación } \\
\text { Integral }\end{array}$ & Marco Ordenador & Definición \\
\hline \multirow{5}{*}{ 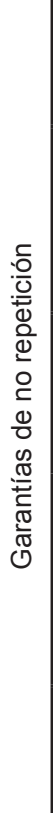 } & Afectación & Fuerza Motriz & $\begin{array}{l}\text { Se debe medir la magnitud del } \\
\text { grado de afectación, la cual, en } \\
\text { función del principio debe ser } \\
\text { tendiente a la baja. }\end{array}$ \\
\hline & Restitución & Presión & $\begin{array}{l}\text { Se mide la presión en función } \\
\text { de los derechos vulnerados } \\
\text { por medio de los medios } \\
\text { legales existentes (demandas, } \\
\text { denuncias, reclamos, quejas, } \\
\text { etc.) }\end{array}$ \\
\hline & Indemnización & Estado & $\begin{array}{l}\text { Se mide como el sistema de } \\
\text { gestión de la deuda ambiental } \\
\text { resarce parte de su deuda a } \\
\text { la comunidad humana como } \\
\text { parte integrante del ambiente. }\end{array}$ \\
\hline & $\begin{array}{l}\text { Medidas de } \\
\text { satisfacción }\end{array}$ & Impacto & $\begin{array}{l}\text { Se mide el grado de aceptación } \\
\text { de la responsabilidad de las o } \\
\text { la componía(s) u organización } \\
\text { dedicada a la actividad } \\
\text { examinada que ha generado } \\
\text { afectaciones y/0 fuentes de } \\
\text { contaminación. }\end{array}$ \\
\hline & $\begin{array}{l}\text { Rehabilitación } \\
\text { (Restauración) }\end{array}$ & Respuesta & $\begin{array}{l}\text { Se mide la magnitud de la } \\
\text { rehabilitación de los sectores } \\
\text { afectados por fuentes de } \\
\text { contaminación. }\end{array}$ \\
\hline
\end{tabular}


Tabla 2. Modelo de indicadores ambientales y sociales propuesto para medir la deuda ambiental

\begin{tabular}{|c|c|c|}
\hline \multicolumn{3}{|c|}{ Afectación } \\
\hline Tipo de indicador & $\begin{array}{l}\text { Ambiental (Contaminación/ } \\
\text { Depredación) }\end{array}$ & $\begin{array}{l}\text { Mide el tipo de problema ambiental: } \\
\text { depredación de recursos o la contaminación } \\
\text { con residuos. }\end{array}$ \\
\hline $\begin{array}{c}\text { Tipo de relación (Interpretada respecto al } \\
\text { aumento) }\end{array}$ & Bucle negativo & $\begin{array}{l}\text { Mantiene al sistema en un estado alejado del } \\
\text { equilibrio. }\end{array}$ \\
\hline Tipo de aspecto (Miller 1978) & Estructura & $\begin{array}{l}\text { Son afectaciones ambientales que generan } \\
\text { influencia en el aumento y disminución de la } \\
\text { deuda ambiental. }\end{array}$ \\
\hline Tipo de actividad (Gállopin, 2001) & Insumo o entrada & $\begin{array}{l}\text { Son la entrada para la generación de la } \\
\text { deuda ambiental (A la vez son producto de } \\
\text { las relaciones entre las actividades petroleras } \\
\text { y el ambiente). }\end{array}$ \\
\hline Tipo según marco ordenador & Fuerza motriz & $\begin{array}{l}\text { Es la primera entrada que impulsa, en } \\
\text { primera instancia, la generación de la deuda } \\
\text { ambiental. }\end{array}$ \\
\hline $\begin{array}{l}\text { Estilo de sustentabilidad (Pieri, 2005) } \\
\text { según el efecto del aumento del indicador }\end{array}$ & Muy débil & $\begin{array}{l}\text { Su aumento indica que hay más preocupación } \\
\text { por parte de las empresas y el Estado de } \\
\text { incrementar el Capital total sin tomar en } \\
\text { cuenta los factores ambientales afectados. }\end{array}$ \\
\hline \multicolumn{3}{|c|}{ Restitución } \\
\hline Tipo de indicador & Social & $\begin{array}{l}\text { Mide el efecto social de las poblaciones por la } \\
\text { afectación ambiental debido a vulneración de } \\
\text { derechos de la naturaleza. }\end{array}$ \\
\hline $\begin{array}{c}\text { Tipo de relación (Interpretada respecto al } \\
\text { aumento) }\end{array}$ & Bucle positivo & $\begin{array}{l}\text { Cambia el estado del sistema de alejado del } \\
\text { equilibrio a un sistema en equilibrio. }\end{array}$ \\
\hline Tipo de aspecto (Miller 1978) & Control & $\begin{array}{l}\text { Son indicadores que influyen en las normas } \\
\text { de decisión del pasivo ambiental. }\end{array}$ \\
\hline Tipo de actividad (Gállopin, 2001) & Interna (estado del sistema) & $\begin{array}{l}\text { Son factores internos del comportamiento de } \\
\text { la deuda ambiental, producto de la afectación } \\
\text { por contaminación o depredación. }\end{array}$ \\
\hline Tipo según marco ordenador & Presión & $\begin{array}{l}\text { Impulsa el pago de la deuda ambiental } \\
\text { por medio de la restitución de derechos } \\
\text { vulnerados. }\end{array}$ \\
\hline $\begin{array}{l}\text { Estilo de sustentabilidad (Pieri, 2005) } \\
\text { según el efecto del aumento del indicador }\end{array}$ & Muy fuerte & $\begin{array}{l}\text { Su aumento indica que hay preocupación por } \\
\text { parte de la población que ha sido vulnerada } \\
\text { sus derechos de incrementar el Capital } \\
\text { Natural tomando en cuenta los factores } \\
\text { ambientales afectados. }\end{array}$ \\
\hline \multicolumn{3}{|c|}{ Medidas de satisfacción } \\
\hline Tipo de indicador & Social & $\begin{array}{l}\text { Mide el efecto social de las empresas y el } \\
\text { Estado por la afectación ambiental. }\end{array}$ \\
\hline $\begin{array}{l}\text { Tipo de relación (Interpretada respecto al } \\
\text { aumento) }\end{array}$ & Bucle positivo & $\begin{array}{l}\text { Mide el grado de interiorización, por parte } \\
\text { del Estado y las empresas, de los conflictos } \\
\text { ambientales generados por las afectaciones } \\
\text { de contaminación o depredación. }\end{array}$ \\
\hline Tipo de aspecto (Miller 1978) & Comportamiento & $\begin{array}{l}\text { Reflejan el comportamiento del Estado y } \\
\text { las empresas en función de las normas y } \\
\text { restricciones impuestas por la presión. }\end{array}$ \\
\hline Tipo de actividad (Gállopin, 2001) & Interna (estado del sistema) & $\begin{array}{l}\text { Reflejan el estado del sistema social respecto } \\
\text { a la afectación ambiental y es una condición } \\
\text { bien definida. }\end{array}$ \\
\hline Tipo según marco ordenador & Estado & $\begin{array}{l}\text { Refleja el estado del sistema social que } \\
\text { impulsa el pago de la deuda ambiental. }\end{array}$ \\
\hline $\begin{array}{l}\text { Estilo de sustentabilidad (Pieri, 2005) } \\
\text { según el efecto del aumento del indicador }\end{array}$ & Débil & $\begin{array}{l}\text { La preocupación del Estado y las Empresas } \\
\text { está en mantener cierto capital natural } \\
\text { en función de posibilidades e intereses } \\
\text { concretos, donde la sustituibilidad de } \\
\text { capitales no es perfecta. }\end{array}$ \\
\hline
\end{tabular}




\begin{tabular}{|c|c|c|}
\hline Tipo de indicador & Social & $\begin{array}{l}\text { Mide el efecto social de las Empresas, } \\
\text { Estado y poblaciones por la afectación } \\
\text { ambiental }\end{array}$ \\
\hline $\begin{array}{l}\text { Tipo de relación (Interpretada } \\
\text { respecto al aumento) }\end{array}$ & Bucle positivo & $\begin{array}{l}\text { Mide el grado de interiorización, por } \\
\text { parte del Estado y las empresas, de los } \\
\text { conflictos ambientales generados por } \\
\text { las afectaciones de contaminación o } \\
\text { depredación. }\end{array}$ \\
\hline Tipo de aspecto (Miller 1978) & Comportamiento & $\begin{array}{l}\text { Reflejan el comportamiento del Estado y } \\
\text { las empresas en función de las normas y } \\
\text { restricciones impuestas por la presión y } \\
\text { el estado del sistema. }\end{array}$ \\
\hline Tipo de actividad (Gállopin, 2001) & Salida & $\begin{array}{l}\text { Son variables que resultan de la } \\
\text { interacción para disminuir la deuda } \\
\text { ambiental. }\end{array}$ \\
\hline Tipo según marco ordenador & Impacto & $\begin{array}{l}\text { Son indicadores que miden el grado de } \\
\text { influencia que tienen las acciones para } \\
\text { disminuir la deuda ambiental y llevar al } \\
\text { sistema un estado de equilibrio. }\end{array}$ \\
\hline $\begin{array}{l}\text { Estilo de sustentabilidad (Pieri, 2005) } \\
\text { según el efecto del aumento del } \\
\text { indicador }\end{array}$ & Fuerte & $\begin{array}{l}\text { La preocupación del sistema social, } \\
\text { Estado, Empresas y poblaciones } \\
\text { vulneradas está en mantener el capital } \\
\text { natural crítico inalterable; capital natural } \\
\text { sostenible, sustituible o renovable } \\
\text { puede ser sustituido por capital humano } \\
\text { manufacturado, financiero y construido. }\end{array}$ \\
\hline & Rehabilitación & \\
\hline Tipo de indicador & Ambiental & $\begin{array}{l}\text { Mide el grado de rehabilitación de la } \\
\text { afectación de los ecosistemas por } \\
\text { contaminación implementando medidas } \\
\text { de remediación (suelo, agua y aire) y } \\
\text { depredación implementando medidas de } \\
\text { restauración (flora y fauna). }\end{array}$ \\
\hline $\begin{array}{l}\text { Tipo de relación (Interpretada } \\
\text { respecto al aumento) }\end{array}$ & Bucle positivo & $\begin{array}{l}\text { Cambia el estado del sistema de alejado } \\
\text { del equilibrio a un sistema en equilibrio } \\
\text { sin deuda ambiental. }\end{array}$ \\
\hline Tipo de aspecto (Miller 1978) & Estructura & $\begin{array}{l}\text { Cambia a los componentes ambientales } \\
\text { constitutivos del pasivo ambiental que } \\
\text { generan influencia o dependencia. }\end{array}$ \\
\hline Tipo de actividad (Gállopin, 2001) & Salida & $\begin{array}{l}\text { Son variables que resultan de la } \\
\text { interacción para disminuir la deuda } \\
\text { ambiental. }\end{array}$ \\
\hline Tipo según marco ordenador & Respuesta & $\begin{array}{l}\text { Son indicadores que miden la respuesta } \\
\text { que genera la interacción total del } \\
\text { sistema cambiando la estructura de } \\
\text { la afectación que genera el pasivo } \\
\text { ambiental. }\end{array}$ \\
\hline $\begin{array}{l}\text { Estilo de sustentabilidad (Pieri, 2005) } \\
\text { según el efecto del aumento del } \\
\text { indicador }\end{array}$ & Muy fuerte & $\begin{array}{l}\text { Su aumento indica que hay preocupación } \\
\text { por parte del Sistema social (Estado, } \\
\text { Empresas y poblaciones afectadas) por } \\
\text { incrementar el capital natural tomando } \\
\text { en cuenta los factores ambientales } \\
\text { afectados y la no sustituibilidad con el } \\
\text { capital humano. }\end{array}$ \\
\hline \multicolumn{3}{|c|}{ Garantías de no repetición } \\
\hline
\end{tabular}




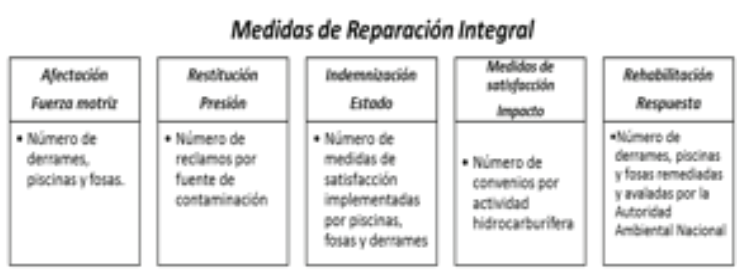

Garanties de no repeticion: miden por el grabo de implementacibo y electividad de las politicas piblicas relacionadas a pasives ambientales

Tabla 3

Número y porcentaje de derrames por provincia.

\begin{tabular}{|l|c|c|}
\hline \multicolumn{1}{|c|}{ Provincia } & $\begin{array}{c}\text { Número de } \\
\text { derrames }\end{array}$ & $\begin{array}{c}\text { Porcentaje de } \\
\text { derrames }\end{array}$ \\
\hline Esmeraldas & 12 & $1,09 \%$ \\
\hline Guayas & 17 & $1,54 \%$ \\
\hline Na9 & 3 & $0,27 \%$ \\
\hline Napo & 15 & $1,36 \%$ \\
\hline Orellana & 448 & $40,62 \%$ \\
\hline Pichincha & 4 & $0,36 \%$ \\
\hline Santa Elena & 178 & $16,14 \%$ \\
\hline Santo domingo de los Tsáchilas & 3 & $0,27 \%$ \\
\hline Sucumbíos & 423 & $38,35 \%$ \\
\hline Total general & $\mathbf{1 1 0 3}$ & $\mathbf{1 0 0 , 0 0 \%}$ \\
\hline
\end{tabular}

Fuente: Sistema de Indicadores de Pasivos Ambientales y Sociales del sector Hidrocarburífero Nacional, 2010

Tabla 4

Número y porcentaje de piscinas por provincia.

\begin{tabular}{|l|c|c|}
\hline \multicolumn{1}{|c|}{ Provincia } & $\begin{array}{c}\text { Número de } \\
\text { piscinas }\end{array}$ & $\begin{array}{c}\text { Porcentaje de } \\
\text { piscinas }\end{array}$ \\
\hline Esmeraldas & 16 & $0,98 \%$ \\
\hline Guayas & 1 & $0,06 \%$ \\
\hline Napo & 12 & $0,74 \%$ \\
\hline Orellana & 688 & $42,34 \%$ \\
\hline Pastaza & 24 & $1,48 \%$ \\
\hline Santa Elena & 21 & $1,29 \%$ \\
\hline Sucumbíos & 862 & $53,05 \%$ \\
\hline Tungurahua & 1 & $0,06 \%$ \\
\hline Total general & 1625 & $100,00 \%$ \\
\hline
\end{tabular}

Fuente: Sistema de Indicadores de Pasivos Ambientales y

Sociales del sector Hidrocarburífero Nacional, 2010

Tabla 5

Número y porcentaje de fosas por provincia (Distrito Amazónico)

\begin{tabular}{|c|c|c|}
\hline PROVINCIA & \multicolumn{1}{|c|}{$\begin{array}{c}\text { NÚMERO DE } \\
\text { FOSAS }\end{array}$} & $\begin{array}{c}\text { PORCENTAJE } \\
\text { DE FOSAS }\end{array}$ \\
\hline ORELLANA & 699 & 100,00 \\
\cline { 1 - 1 } SUCUMBIOS & & \\
\hline Total general & 699 & $100,00 \%$ \\
\hline
\end{tabular}

Fuente: Sistema de Indicadores de Pasivos Ambientales y Sociales del sector Hidrocarburífero Nacional, 2010

\section{Conclusión}

La deuda o pasivo ambiental se genera por la sobreexplotación de los servicios ambientales contaminado a los ecosistemas. Para remediar la deuda ambiental generada por el desarrollo del sistema socio ecológico, la aplicación del principio de reposición integral debe ser extendida al componente biofísico, sin olvidar el componente ético sobre el respeto a los derechos de la naturaleza y de las especies a vivir en armonía.

Las propuestas en el sector hidrocarburífero, en base a las estadísticas generadas por el PRASS, son los indicadores propuestos con esta metodología.

El estado del pago de la deuda ambiental con respecto a las actividades hidrocarburíferas del 2013, según el modelo propuesto, muestra que en el Ecuador falta implementar muchas medidas del principio de reparación integral social y ambiental.

\section{Referencias bibliográficas}

Aguirre Royuela, M. Á. (2002). Los sistemas de indicadores ambientales y su papel en la información e integración del medio ambiente. En T. Y. Civil, \& C. Y. Colegio De Ingenieros De Caminos (Ed.), I Congreso de Ingeniería Civil, Territorio y Medio Ambiente (Primera edición ed., Vol. I, págs. 1231 - 1256). Madrid, España. Recuperado el 2015 de 06 de 03, de http://www.ciccp.es/webantigua/icitema/Comunicaciones/Tomo_II/ T2p1231.pdf

Beristain, C. M., Páez Rovira, D., \& Fernández, I. (2009). Las palabras de la selva Estudio psicosocial del impacto de las explotaciones petroleras de Texaco en las comunidades amazónicas de Ecuador (1 ed., Vol. 1). (hegoa, Ed.) País Vasco, España: Instituto de Estudios sobre Desarrollo y cooperación Internacional Universidad del Paíss Vasco.

Guttman, S. E., Zorro, S. C., Cuervo de Forero, A., \& Ramírez J., C. J. (julio 2004). Diseño de un sistema de indicadores para el Distrito Capital de Bogotá (Vol. Disponible en: http://www.cepal.org ó http://www.eclac. org). (C.-P. Col/01/002, Ed.) Santiago de Chile, Región Metropolitana de Santiago, Serie Estudios y Perspectivas 3, Chile: Naciones Unidas.

IGM, Senplades. (2013). El medio ambiente. En S. IGM, \& IGM (Ed.), Atlas geográfico de la República del Ecuador (Segunda Edición ed., Vol. II, págs. 243-308). Quito, Pichincha, Ecuador: IGM. Obtenido de http:// www.geoportaligm.gob.ec/portal/index.php/geoeduca/ Atlas-Nacional-del-Ecuador/atlas-geografico-nacional-del-Ecuador-2013. 


\begin{tabular}{|c|c|}
\hline \multicolumn{2}{|r|}{ OLOGIA PROP } \\
\hline Nombre del indicador & Número de derrames, piscinas y fosas. \\
\hline Definición & $\begin{array}{l}\text { Es el número de derrames ocurridos, piscinas y fosas generadas } \\
\text { en el sistema hidrocarburífero registrados, para un período } \\
\text { determinado y unidad de análisis. }\end{array}$ \\
\hline \multicolumn{2}{|r|}{ Fórmula de cálculo } \\
\hline \multicolumn{2}{|c|}{ Número de derrames, piscnas y fosas $=\sum_{i=0}^{n}$ Derrames $_{i}+$ Piscinas $_{i}+$ Fosas $_{i}$} \\
\hline \multicolumn{2}{|r|}{ Definicion de las variables relacionadas } \\
\hline \multicolumn{2}{|c|}{$\begin{array}{l}\text { Derrame: Consiste en la liberación de hidrocarburos y/o derivados al ambiente; se produce por causas } \\
\text { operacionales, atentados o eventos naturales. } \\
\text { Piscina: según el SIPAS HN una piscina es una excavación, parte del proceso de extracción de petróleo del } \\
\text { subsuelo. Sirven como sitio para la disposición de desechos químicos, lodos, ripios de perforación y pruebas } \\
\text { de producción de las distintas actividades de perforación y producción. } \\
\text { Fosa: Como se menciona en el PRIPA (2012) una fosa es una excavación de dimensiones pequeñas, en } \\
\text { promedio ( } 2 \mathrm{~m} \times 2 \mathrm{~m} \times 3 \mathrm{~m}) \text {, que se realizaba como mala práctica cerca de las orillas de los ríos, lagunas o } \\
\text { pantanos con la finalidad de confinar el material contaminado producto de los derrames de hidrocarburos. }\end{array}$} \\
\hline \multicolumn{2}{|r|}{ Metodologia de cálculo } \\
\hline \multicolumn{2}{|c|}{$\begin{array}{l}\text { Para calcular este indicador se realiza la sumatoria de todos los derrames ocurridos, piscinas y fosas } \\
\text { generadas durante las operaciones de la actividad hidrocarburífera. }\end{array}$} \\
\hline \multicolumn{2}{|r|}{ Limitaciones técnicas } \\
\hline \multicolumn{2}{|c|}{$\begin{array}{l}\text { Solo se registran los derrames que tienen un volumen mayor a } 5 \text { barriles, de acuerdo a lo establecido por el } \\
\text { Reglamento Ambiental para Operaciones Hidrocarburíferas (rango mínimo para reportar el evento). En } 735 \\
\text { registros de derrames no se dispone de toda la información referente al estado actual del derrame con } \\
\text { respecto a la remediación, de igual manera } 310 \text { registros no tienen la información de la causa del derrame. } \\
\text { Aunque la información disponible ha sido depurada y contrastada, se debe señalar que, al igual que en otros } \\
\text { indicadores, los datos presentan vacíos, subregistros e inconsistencias. Evidentemente estos problemas han } \\
\text { sido minimizados al máximo mediante el proceso de depuración de las bases de datos. En este sentido, no } \\
\text { se debe olvidar que el indicador trabaja sobre la información existente y disponible, siendo ese su alcance. } \\
\text { Para este caso en particular, } 262 \text { piscinas registradas en el SIPAS, no disponen de toda la información } \\
\text { referente al estado actual de la piscina con respecto a la remediación. } \\
\text { El dato de número de fosas corresponde únicamente a las registradas en las provincias de Orellana y } \\
\text { Sucumbíos (operación estatal). } \\
\text { No se tiene información sobre los campos de la costa ni de la fase de comercialización. } \\
\text { Otra limitación es que al tomar en cuenta solo datos y no magnitud de derrames, fosas y piscinas se puede } \\
\text { interpretar erróneamente que en un año hubo pocas fuentes de contaminación, pero como el caso del } \\
\text { derrame de } 1987 \text { fue un evento que sucedió por la rotura de } 40 \mathrm{~km} \text { del SOTE cuyos efectos ambientales } \\
\text { permanecen hasta la época actual, esto es una limitación del indicador. }\end{array}$} \\
\hline $\begin{array}{l}\text { Unidad de medida o expresión } \\
\text { del indicador }\end{array}$ & Número \\
\hline Interpretación del indicador & $\begin{array}{l}\text { Es un indicador ambiental de contaminación debido a que mide el } \\
\text { total de eventos en forma de derrames, piscinas y fosas que afectan a } \\
\text { las cavidades ambientales suelo, agua y aire introduciendo } \\
\text { hidrocarburos y derivados. El tipo de relación es de bucle de } \\
\text { realimentación negativa debido a que su aumento tiende a mantener } \\
\text { al sistema alejado del equilibrio, es decir en deuda. Conforman la } \\
\text { estructura de la deuda ambiental debido a que su aumento genera } \\
\text { relaciones de influencia en el mantenimiento de la misma. Son } \\
\text { insumos ya que son la entrada para la generación de la deuda. Se lo } \\
\text { ha catalogado como fuerza motriz, por ser el impulsor de la generación } \\
\text { de la deuda ambiental hidrocarurífera. De estilo de sustentabilidad } \\
\text { muy débil ya que su aumento, indica que la preocupación de las } \\
\text { empresas de hidrocarburos y el Estado reside en aumentar el capital }\end{array}$ \\
\hline
\end{tabular}


ONU-CEPAL. (abril 2010). El Desarrollo Sostenible en América Latina y el Caribe: tendencias, avances y desafíos en materia de consumo y producción sostenibles, minería, transporte, productos químicos y gestión de residuos; (Primera edición ed.). Santiago de Chile, Región Metropolitana de Santiago, Chile: Naciones Unidas.

PNUMA Oficina Regional para América Latina y el Caribe. (2008). GEO Ecuador 2008, informe sobre el estado del medio ambiente. Quito, Pichincha, Ecuador: PNUMA-FLACSO ECUADOR-MINISTERIO DEL AMBIENTE-BID-ECORAE-PMRC-ESPOL.

PRAS-MAE (SINARI). (Agosto, 2014). Sistema de Información Nacional de la Reparación Integral de los pasivos socio-ambientales (SINARI) (Primera edición ed., Vol. 2). (V. preliminar, Ed.) Quito, Pichincha, Ecuador: PRAS-MAE.

Quiroga, M. R. (julio 2009). Guía metodológica para desarrollar indicadores ambientales y de desarrollo sostenible en países de América Latina y el Caribe, CEPAL. (Primera ed., Vols. Manuales, Serie 61; Disponible en: http://www.cepal.org/ o http://www.eclac.org). Santiago de Chile, Región Metropolitana de Chile, Chile: CEPAL, PNUD, Naciones Unidas.

Senplades. (2013). Plan Nacional para el Buen Vivir 2013-2017 (Tercera edición ed.). (R. Correa Delgado, J. Glas, P. Muñoz, C. Vaca, G. Long, H. Arellano, . . A. M. Larrea, Edits.) Quito, Pichincha, Ecuador: El Telégrafo, UMA Creativa. Obtenido de www.buenvivir.gob.ec

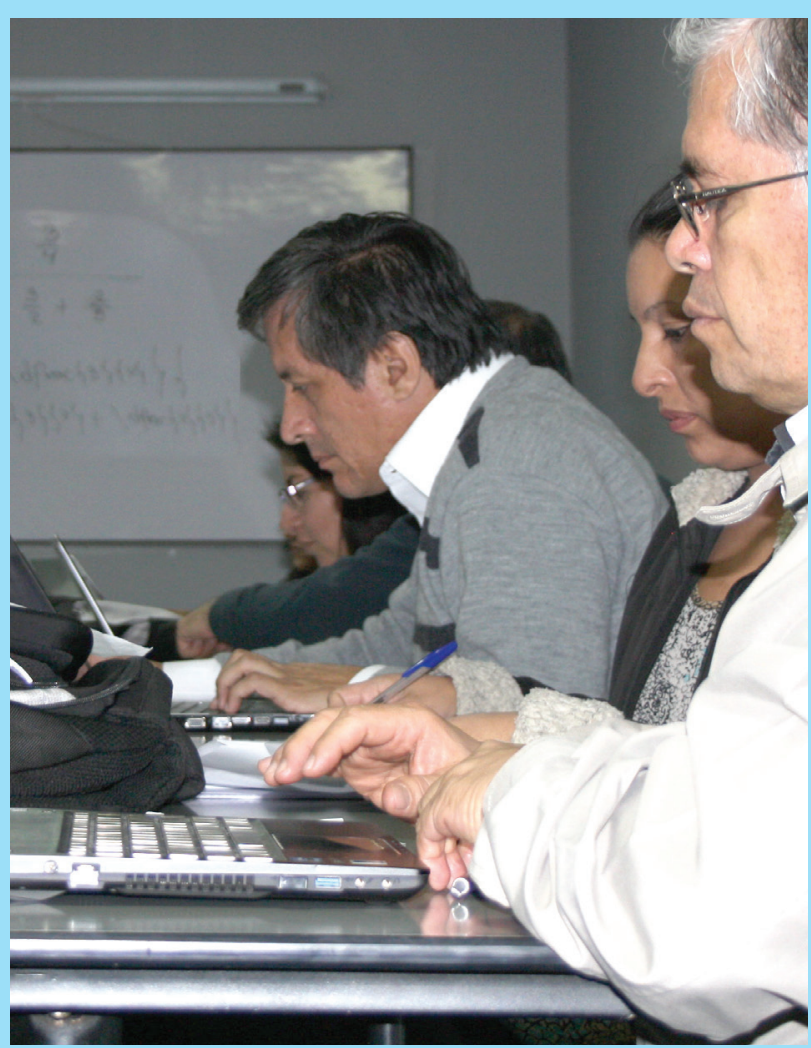

LA FIGEMPA FORTALECE CAPACITACIÓN Y ACTUALIZACIÓN
PROFESIONAL DE SUS DOCENTES

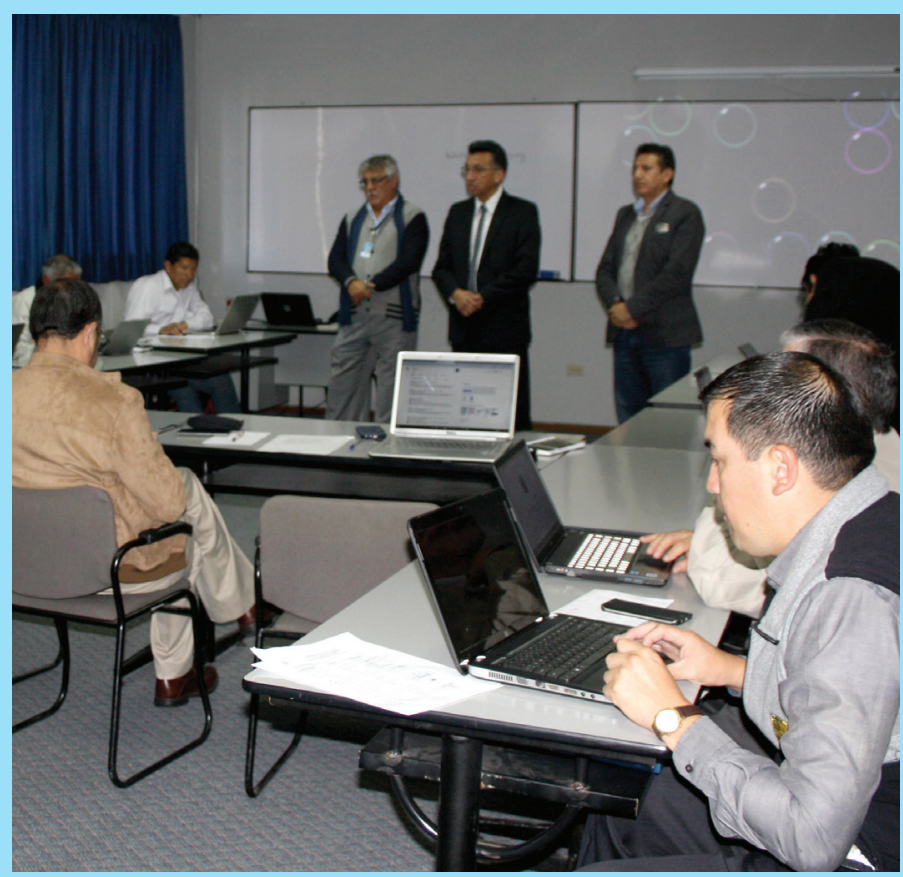

Con el objetivo de fortalecer el desempeño académico, el desarrollo integral y la formación profesional de los docentes de la Facultad de Ingeniería en Geología, Minas, Petróleos y Ambiental, así como reconocer el derecho de los profesores a recibir una capacitación periódica acorde a su formación profesional y la cátedra que imparte, que fomente e incentive la superación personal y pedagógica, la FIGEMPA, por intermedio de su Asociación de Profesores, con el aval de la Dirección de Desarrollo Académico, y en el marco del Plan de Capacitación Docente aprobado por Consejo Directivo de la Facultad, han llevado a cabo los siguientes cursos: Recursos Tecnológicos para la Docencia Universitaria, del 12 al 16 de septiembre de 2016; Metodología de Trabajo en el Aula, del 19 al 23 de septiembre de 2016; Redacción Científica del 26 al 30 de septiembre de 2016; Formulación de Proyectos de Investigación del 20 al 24 de febrero de 2017; Manejo de Blogs Académicos; y, Redacción de Textos con LATEX, del 21 al 24 de marzo de 2017, con una duración de 40 horas. Se espera completar 400 horas para aquellos profesores que asistan a todos los eventos.

Este esfuerzo de perfeccionamiento docente y que sin lugar a duda ayudará en el proceso de formación de nuestros estudiantes, se ha desarrollado aprovechando la fortaleza y capacidades de los propios recursos humanos existentes en la Institución como son: Susana Cadena de la DTIC, Danilo García del Comité de Ética, Bertha Estrella y Fernando Salazar de la Coordinación de Investigación Formativa, Marco González y Ramiro Guerrón de la FIGEMPA, de funcionarios de la actual Dirección de Desarrollo Académico y de la Asociación de Profesores, entre otros. 